\title{
Terrestrial deuterium-to-hydrogen ratio in water in hyperactive comets
}

\author{
Dariusz C. Lis ${ }^{1,2}$, Dominique Bockelée-Morvan ${ }^{3}$, Rolf Güsten ${ }^{4}$, Nicolas Biver ${ }^{3}$, Jürgen Stutzki ${ }^{5}$, Yan Delorme ${ }^{2}$, \\ Carlos Durán ${ }^{4}$, Helmut Wiesemeyer ${ }^{4}$, and Yoko Okada ${ }^{5}$ \\ 1 Jet Propulsion Laboratory, California Institute of Technology, 4800 Oak Grove Drive, Pasadena, CA 91109, USA \\ e-mail: Dariusz.C.Lis@jpl.nasa.gov \\ 2 Sorbonne Université, Observatoire de Paris, Université PSL, CNRS, LERMA, 75005 Paris, France \\ 3 LESIA, Observatoire de Paris, Université PSL, CNRS, Sorbonne Université, Université Paris Diderot, Sorbonne Paris Cité, \\ 5 place Jules Janssen, 92195 Meudon, France \\ 4 Max-Planck-Institut für Radioastronomie, Auf dem Hügel 69, 53121 Bonn, Germany \\ 5 I. Physikalisches Institut, Universität zu Köln, Zülpicher Straße 77, 50937 Köln, Germany \\ Received 26 March 2019 / Accepted 11 April 2019
}

\begin{abstract}
The $\mathrm{D} / \mathrm{H}$ ratio in cometary water has been shown to vary between 1 and 3 times the Earth's oceans value, in both Oort cloud comets and Jupiter-family comets originating from the Kuiper belt. This has been taken as evidence that comets contributed a relatively small fraction of the terrestrial water. We present new sensitive spectroscopic observations of water isotopologues in the Jupiterfamily comet 46P/Wirtanen carried out using the GREAT spectrometer aboard the Stratospheric Observatory for Infrared Astronomy (SOFIA). The derived D/H ratio of $(1.61 \pm 0.65) \times 10^{-4}$ is the same as in the Earth's oceans. Although the statistics are limited, we show that interesting trends are already becoming apparent in the existing data. A clear anti-correlation is seen between the $\mathrm{D} / \mathrm{H}$ ratio and the active fraction, defined as the ratio of the active surface area to the total nucleus surface. Comets with an active fraction above 0.5 typically have $\mathrm{D} / \mathrm{H}$ ratios in water consistent with the terrestrial value. These hyperactive comets, such as $46 \mathrm{P} /$ Wirtanen, require an additional source of water vapor in their coma, explained by the presence of subliming icy grains expelled from the nucleus. The observed correlation may suggest that hyperactive comets belong to a population of ice-rich objects that formed just outside the snow line, or in the outermost regions of the solar nebula, from water thermally reprocessed in the inner disk that was transported outward during the early disk evolution. The observed anti-correlation between the active fraction and the nucleus size seems to argue against the first interpretation, as planetesimals near the snow line are expected to undergo rapid growth. Alternatively, isotopic properties of water outgassed from the nucleus and icy grains may be different due to fractionation effects at sublimation. In this case, all comets may share the same Earth-like D/H ratio in water, with profound implications for the early solar system and the origin of Earth's oceans.
\end{abstract}

Key words. comets: general - comets: individual: 46P/Wirtanen - submillimeter: planetary systems - astrochemistry Kuiper belt: general

\section{Introduction}

One of the key questions for modern astrophysics and planetary science concerns the development of the conditions of habitability in planetary systems, such as the early protosolar nebula. Water, an essential ingredient for carbon-based life as we know it (Westall 2018), is formed primarily via surface reactions in icy mantles of interstellar dust grains (the gas-phase chemistry only becomes efficient at temperatures above $\sim 300 \mathrm{~K}$ ). These grains subsequently find their way through dense protostellar cores to protoplanetary disks, where they are partially processed thermally in the warm inner disk before being locked up in small bodies such as comets or asteroids (van Dishoeck et al. 2014).

In the standard model of the protosolar nebula, the temperature in the terrestrial planet forming zone was too high for water ice to survive. Consequently, the Earth accreted dry and the present-day water would have been delivered in a later phase, together with organics, by external sources such as comets or asteroids (O'Brien et al. 2018). An alternative explanation is in situ, early delivery of Earth's water, either incorporated into olivine grains or through the oxidation of an early hydrogen atmosphere by $\mathrm{FeO}$ in the terrestrial magma ocean, both of which may have contributed to some degree (see O'Brien et al. 2018 and references therein).

The $\mathrm{D} / \mathrm{H}$ ratio provides key constraints on the origin and thermal history of water molecules. Deuterium was produced in the Big Bang, with an abundance of about $2.5 \times 10^{-5}$ with respect to hydrogen (Cooke et al. 2014). The reference protosolar $\mathrm{D} / \mathrm{H}$ ratio in hydrogen is $2.1 \times 10^{-5}$, which is close to the Big Bang value (Geiss \& Gloeckler 1998). However, in the cold, dense, CO-depleted interstellar medium, deuterium atoms are preferentially sequestered in heavy molecules due to differences in zeropoint vibrational energies (Ceccarelli et al. 2014). Consequently, the $\mathrm{D} / \mathrm{H}$ ratio in heavy molecules may be enhanced by orders of magnitude, and doubly or even triply deuterated species have been detected (Lis et al. 2002; Parise et al. 2004). Deuteration in water is less extreme than in other molecules, with water $\mathrm{D} / \mathrm{H}$ ratios of order $0.001-0.01$ typically measured in low-mass 
protostars similar to our Sun (Ceccarelli et al. 2014). Subsequent isotopic exchanges between water molecules and molecular hydrogen in the warm inner disk drives the ratio back toward the protosolar value (Drouart et al. 1999).

The highest solar system $\mathrm{D} / \mathrm{H}$ ratios in water, about $7.3 \times 10^{-4}$ measured in LL3 matrix clays or $\mathrm{R}$ chondrites (Deloule et al. 1998; Alexander et al. 2012; McCanta et al. 2008), are close to the interstellar medium values. The $\mathrm{D} / \mathrm{H}$ ratio in Earth's ocean water, the Vienna Standard Mean Ocean Water (VSMOW), is significantly lower, $(1.5576 \pm 0.0001) \times$ $10^{-4}$, although still enhanced with respect to the protosolar ratio in hydrogen. How representative this value is for the bulk of Earth's water is a subject of discussion in the light of recent measurements of a low $\mathrm{D} / \mathrm{H}$ ratio in deep mantle materials (Hallis 2015). Currently, carbonaceous chondrites, in particular $\mathrm{CI}$ and $\mathrm{CM}$ types, appear to best match the terrestrial $\mathrm{D} / \mathrm{H}$ ratio (Alexander et al. 2012).

Comets are the most primitive volatile-rich bodies in the solar system. The $\mathrm{D} / \mathrm{H}$ ratio has been measured in a handful of Oort cloud comets, with typical values of about twice VSMOW (Bockelée-Morvan et al. 2015). The Herschel Space Observatory provided the first measurements of the $\mathrm{D} / \mathrm{H}$ ratio in two Jupiter-family comets, 103P/Hartley (Hartogh et al. 2011) and 45P/Honda-Mrkos-Pajdušáková (Lis et al. 2013), both consistent with VSMOW. A relatively high $\mathrm{D} / \mathrm{H}$ ratio, three times VSMOW, was subsequently measured by Rosetta in another Jupiter-family comet 67P/Churyumov-Gerasimenko (Altwegg et al. 2015). The VSMOW D/H value measured in the Oort cloud comet C/2014 Q2 (Lovejoy, Biver et al. 2016) suggests that the same isotopic diversity is present in the two comet families.

The large variations in the $\mathrm{D} / \mathrm{H}$ ratio in cometary water have been interpreted as reflecting their formation in different regions of the solar nebula. Models considering isotopic exchanges in an evolving accretion disk predict an increase in the $\mathrm{D} / \mathrm{H}$ ratio with increasing distance from the star (Drouart et al. 1999). The same isotopic diversity observed in both Oort cloud and Jupiter-family comets could then be explained by the recent evidence that the formation zones of the two families largely overlapped and extended over a broad range of heliocentric distances (Brasser \& Morbidelli 2013).

In this Letter we present a new measurement of the $\mathrm{D} / \mathrm{H}$ ratio in the Jupiter-family comet 46P/Wirtanen carried out using the Stratospheric Observatory for Infrared Astronomy (SOFIA). This comet, which was the initial target of the Rosetta mission, has an orbital period of $5.439 \mathrm{yr}$ and made a close approach to Earth (0.08 au) a few days after its perihelion passage on 2018 December 12 at 22:20 UT (perihelion distance $q=1.05536 \mathrm{au}$ ). Comet 46P/Wirtanen belongs to the category of hyperactive comets, emitting more water molecules than can be expected given the size of the nucleus, which is explained by the presence of sublimating water-ice-rich particles within the coma. Using a sample of comets with known $\mathrm{D} / \mathrm{H}$ ratios in water and nucleus sizes, we show that a remarkable correlation is present between the $\mathrm{D} / \mathrm{H}$ ratio and hyperactivity.

\section{SOFIA observations of comet 46P/Wirtanen}

Previous spectroscopic detections of HDO were obtained from observations of ro-vibrational and rotational transitions in the infrared and submillimeter domains (Bockelée-Morvan et al. 2015). Low-energy rotational transitions of water are not accessible from the ground or suborbital platforms. However, the atmosphere at stratospheric altitudes is sufficiently transparent at the frequencies of water isotopologues. In particular, the 547 and $509 \mathrm{GHz} 1_{1,0}-1_{0,1}$ transitions of $\mathrm{H}_{2}^{18} \mathrm{O}$ and $\mathrm{HDO}$, previously observed in several comets by Herschel, are now accessible from SOFIA and can be used to accurately measure the $\mathrm{D} / \mathrm{H}$ isotopic ratio. This requires assumptions about the ${ }^{16} \mathrm{O} /{ }^{18} \mathrm{O}$ isotopic ratio, which has been shown to be relatively uniform in comets, $500 \pm 50$, and close to the terrestrial ratio (Bockelée-Morvan et al. 2015).

The close 2018 December apparition of comet 46P/Wirtanen provided an excellent opportunity to demonstrate the utility of SOFIA for $\mathrm{D} / \mathrm{H}$ measurements. Observations presented here were carried out during five SOFIA flights between 2018 December 14 and 20 UT. During each flight, comet Wirtanen was observed in a single flight leg of about 3 hours (the longest time allowed by the flight planning). A typical observing sequence consisted of a 7-17 min on-source integration at the frequency of the $1_{1,0}-1_{0,1}$ transition of $\mathrm{H}_{2}^{18} \mathrm{O}$, followed by a 26-34 min on-source integration at the frequency of the $1_{1,0}-1_{0,1}$ transition of HDO. Monitoring the $\mathrm{H}_{2}^{18} \mathrm{O}$ emission was important for averaging out possible variations in the water production rate during the period of the observations. Additional observational details are provided in Appendix A.1.

Average spectra of the $\mathrm{H}_{2}^{18} \mathrm{O}$ and HDO transitions are shown in Fig. 1. The integrated $\mathrm{H}_{2}^{18} \mathrm{O}$ line intensity is $305 \pm$ $20 \mathrm{mK} \mathrm{km} \mathrm{s}^{-1}$ on the main beam brightness temperature scale ( $15.3 \sigma$, average of all observations). The corresponding integrated line intensity of the HDO emission is $27 \pm 8.8 \mathrm{mK} \mathrm{km} \mathrm{s}^{-1}$ $(3.1 \sigma)$. The resulting $\mathrm{HDO} / \mathrm{H}_{2}^{18} \mathrm{O}$ line intensity ratio is $0.089 \pm$ 0.034 , compared to $0.094 \pm 0.009$ in comet $103 \mathrm{P} /$ Hartley (Hartogh et al. 2011). To model the water isotopologue emission, we used a cometary excitation model similar to that previously applied to Herschel observations (Hartogh et al. 2011; Lis et al. 2013), and assumed a ${ }^{16} \mathrm{O} /{ }^{18} \mathrm{O}$ isotopic ratio of 500 (see Appendix A.2). The resulting $\mathrm{D} / \mathrm{H}$ ratio in water is $(1.61 \pm 0.65) \times 10^{-4}$, where the uncertainty includes statistical, calibration, modeling, and ${ }^{16} \mathrm{O} /{ }^{18} \mathrm{O}$ isotopic ratio uncertainties, combined in quadrature. Comet $46 \mathrm{P} /$ Wirtanen is thus the third Jupiter-family comet with a D/H ratio consistent with the Earth's ocean value.

\section{Correlation between the $\mathrm{D} / \mathrm{H}$ ratio and hyperactivity}

When both the water production rate and the nucleus size are known, it is possible to compute the active fractional area of the nucleus (or active fraction) by dividing the active area by the total nucleus surface. Comets with high active fractions are referred to as hyperactive comets. This hyperactivity requires an additional source of water vapor, explained by the presence of subliming icy grains in the coma that have been expelled from the nucleus. The archetype of a hyperactive comet is $103 \mathrm{P} /$ Hartley, studied by the Deep Impact spacecraft, for which both icy grains and water overproduction were observed (Protopapa et al. 2014; Kelley et al. 2015, 2013). Interestingly, the three Jupiter-family comets with a terrestrial $\mathrm{D} / \mathrm{H}$ ratio, 46P, $103 \mathrm{P}$, and 45P, all belong to the category of hyperactive comets. We therefore investigated quantitatively how the $\mathrm{D} / \mathrm{H}$ ratio correlates with the active fraction using a sample of comets from the literature.

The active fraction was computed using a sublimation model and water production rates derived from Lyman- $\alpha$ observations by the Solar Wind Anisotropies (SWAN) instrument aboard the Solar and Heliocentric Observatory (SOHO; Combi et al. 2019) 


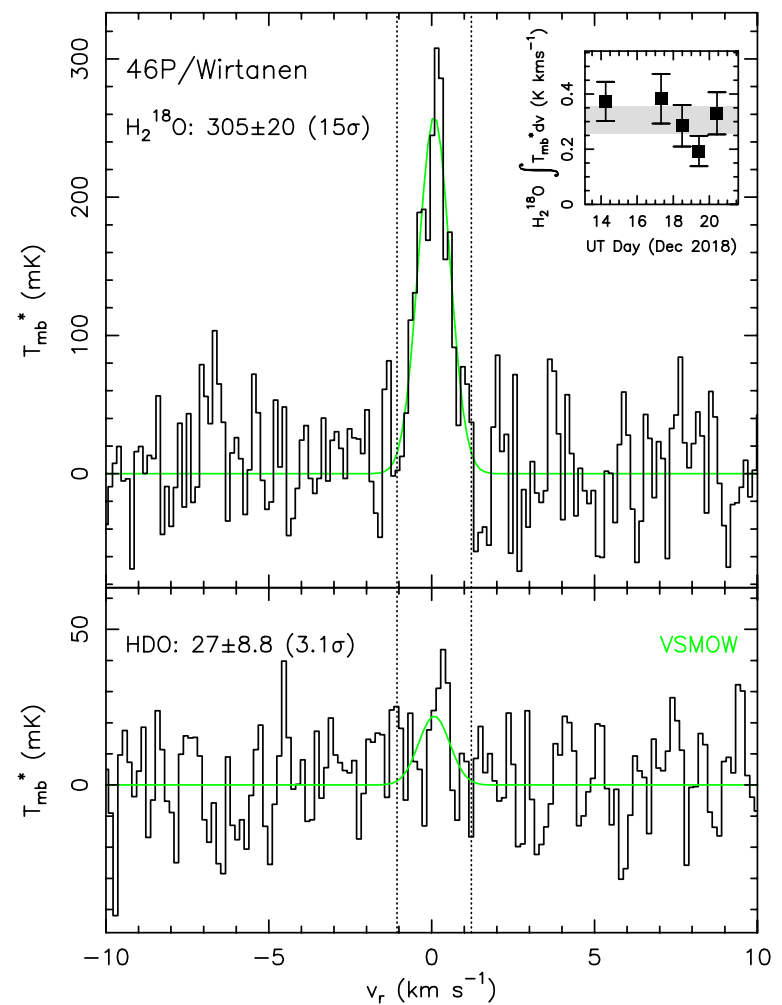

Fig. 1. Spectra of the water isotopologues in comet 46P/Wirtanen. The $1_{1,0}-1_{0,1} \mathrm{H}_{2}^{18} \mathrm{O}$ and HDO transitions are shown in the upper and lower panels, respectively. The intensity scale is the main beam brightness temperature. The spectral resolution is $0.24 \mathrm{MHz}$, corresponding to approximately $0.14 \mathrm{~km} \mathrm{~s}^{-1}$. A Gaussian fit to the $\mathrm{H}_{2}^{18} \mathrm{O}$ spectrum (green line, upper panel) gives a line center velocity $v_{0}=0.08 \pm 0.04 \mathrm{~km} \mathrm{~s}^{-1}$ and a full width at half maximum line width $\Delta v=1.09 \pm 0.09 \mathrm{~km} \mathrm{~s}^{-1}$. Vertical dotted lines indicate the velocity range used in computations of the integrated line intensities $\left(-1.04\right.$ to $\left.1.2 \mathrm{~km} \mathrm{~s}^{-1}\right)$. The green line in the lower panel shows the expected HDO line intensity assuming $\mathrm{D} / \mathrm{H}$ equal to VSMOW. The inset in the upper panel shows the evolution of the $\mathrm{H}_{2}^{18} \mathrm{O}$ integrated line intensity as a function of UT time. Error bars include statistical and calibration uncertainties, combined in quadrature, and the gray shaded area shows the corresponding uncertainty on the average $\mathrm{H}_{2}^{18} \mathrm{O}$ line intensity (ensemble average).

(see Appendix A.3). Since the SWAN field of view is large, water production rates include direct production from the nucleus surface and from subliming icy grains. We computed the active fraction using both production rates at $1 \mathrm{au}$ and at perihelion.

In the sample of comets with D/H determinations (or significant upper limits), only eight comets have a known nucleus size, most of them from spacecraft images or radar measurements (Appendix A.3): 1P/Halley, 8P/Tuttle, 45P/Honda-MrkosPajdušáková, 46P/Wirtanen, 67P/Churyumov-Gerasimenko, 103P/Hartley, C/1996 B2 (Hyakutake), and C/1995 O1 (HaleBopp). We also consider the hyperactive comet C/2009 P1 (Garradd), whose nucleus effective radius has been constrained to be $<5.6 \mathrm{~km}$ (Boissier et al. 2013). The effective nucleus radius of comet $46 \mathrm{P} /$ Wirtanen is estimated to $0.63 \mathrm{~km}$ from radar imaging ${ }^{1}$.

Figure 2 shows a striking anti-correlation between the D/H ratio and the active fraction computed at perihelion. The same trend for a $\mathrm{D} / \mathrm{H}$ ratio decreasing towards the telluric value with increasing active fraction is observed when using the active frac-

\footnotetext{
1 https://uanews.arizona.edu/story/ua-researchercaptures-rare-radar-images-comet-46pwirtanen
}

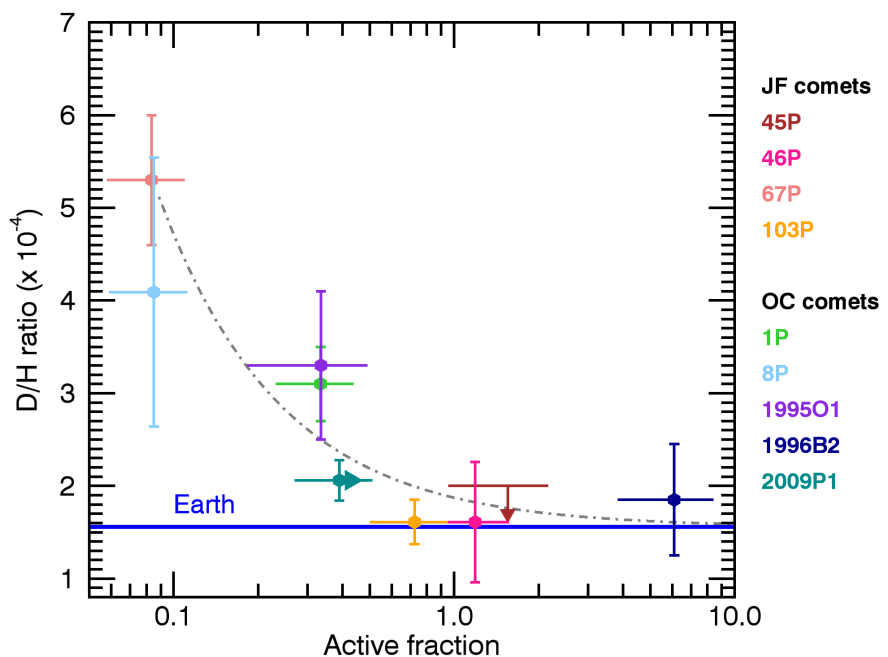

Fig. 2. D/H ratio in cometary water as a function of the active fraction computed from the water production rates measured at perihelion. The uncertainties on the active fraction (horizontal error bars) include a $30 \%$ uncertainty on the water production rates (Combi et al. 2019) and the uncertainty on the nucleus size. The color of each symbol indicates a comet; see legend at right, where the dynamical class is also indicated: Oort cloud (OC) or short-period Jupiter-family (JF) comets. The blue horizontal line corresponds to the VSMOW D/H value. The upper limit for the $\mathrm{D} / \mathrm{H}$ ratio in comet $45 \mathrm{P}$ is indicated by a downward arrow and the lower limit for the active fraction in comet 2009P1 by a right arrow. The dash-dotted line shows the expected $\mathrm{D} / \mathrm{H}$ assuming two sources of water: D-rich $(3.5 \times$ VSMOW) from the nucleus and D-poor (VSMOW). Comets with an active fraction equal to 0.08 are assumed to release only D-rich water.

tion at 1 au from the Sun (Fig. A.1). Values for the D/H ratios are taken from the review of Bockelée-Morvan et al. (2015), except for comet C/1996B2 (Hyakutake), for which we use a revised value of $(1.85 \pm 0.6) \times 10^{-4}$ (Appendix A.4). This long-period comet displayed outbursts and fragmentation events over a few months before and after perihelion, when it released icy grains and chunks, hence the large active fraction (Fig. 2; Combi et al. 2005). The $\mathrm{D} / \mathrm{H}$ ratio reported by Bockelée-Morvan et al. (1998) of $(2.9 \pm 1.0) \times 10^{-4}$ was measured during an outburst, with a large uncertainty mainly related to the scatter in reported water production rates. For this new evaluation, we used updated $Q\left(\mathrm{H}_{2} \mathrm{O}\right)$ values (Combi et al. 2005).

We investigated the processes responsible for the excess of icy grains in hyperactive comets by considering a sample of 18 comets with determined nucleus sizes and water production rates at perihelion (Appendix A.3). As shown in Fig. 3, hyperactivity is not observed for comets with effective nucleus radii larger than $1.2 \mathrm{~km}$ (12 comets in our sample), whereas comets with smaller nuclei, though underrepresented considering the size distribution of cometary nuclei (Fernández et al. 2013), are all hyperactive. This suggests that the large amount of subliming icy aggregates or chunks in hyperactive comets is not related to a higher ice/refractory content. A comparison between the well-studied comets $67 \mathrm{P}$ and $103 \mathrm{P}$ shows that even though the nucleus gas production is much lower in 103P than in $67 \mathrm{P}$, owing to a smaller nucleus size (Fig. 3), the mass loss rate in chunks is larger for 103P (Fulle et al. 2019), thereby explaining its hyperactivity. Estimates of the refractory-to-ice mass ratio in 67P (Herique et al. 2016; Pätzold et al. 2019; Fulle et al. 2019) converge to values between $\delta=3$ and 7, matching the rough estimate of $\delta=3$ for 103P (Fulle et al. 2019). 


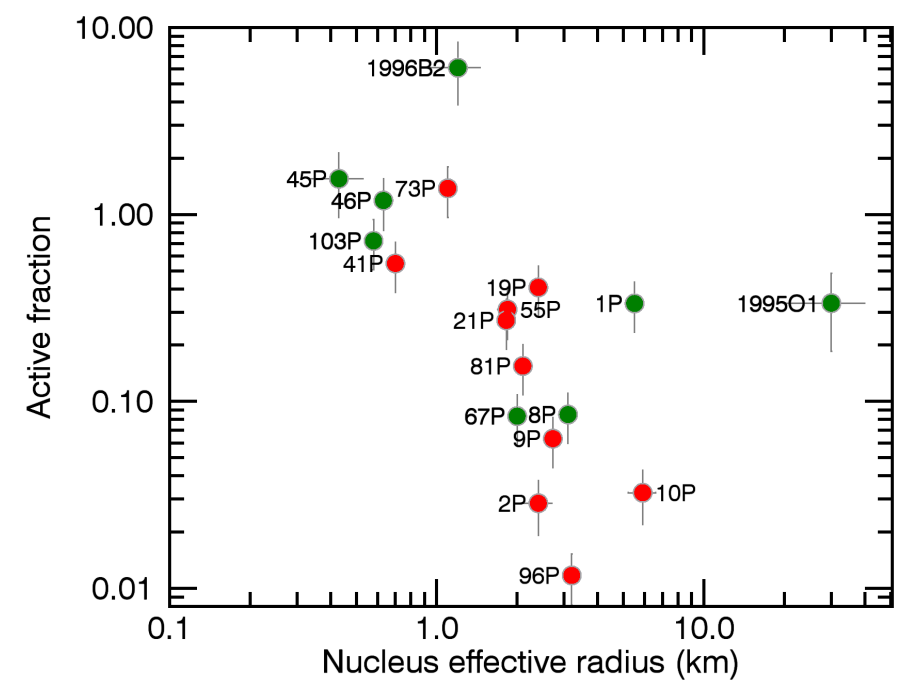

Fig. 3. Active fraction at perihelion as a function of the nucleus size for a sample of 18 comets. The uncertainties in the active fraction (vertical error bars) include a $30 \%$ uncertainty on the water production rates (Combi et al. 2019) and the uncertainty on the nucleus size. The horizontal error bars show the uncertainties in the nucleus size. Green symbols refer to comets for which the $\mathrm{D} / \mathrm{H}$ ratio in water has been measured.

Dust aggregates can be ejected by the sublimation of water ice if the gas pressure overcomes the tensile strength and gravitational pressure of the covering dust layer, which depend on the local gravity, hence nucleus size (Bischoff et al. 2019). However, it remains to be demonstrated why small nuclei eject chunks so efficiently.

\section{Discussion}

Under the hypothesis that hyperactive comets belong to a population of ice-rich comets, their Earth-like D/H ratio would be consistent with their formation in the protoplanetary disk just outside the snow line where a large enhancement in the ice surface density is expected, thus favoring planetesimal formation (Schoonenberg \& Ormel 2017). This would explain the surprising result from Rosetta that ice-poor, D-rich comets, such as comet $67 \mathrm{P}$, are less rich in water than material from carbonaceous meteorites formed closer to the Sun (Fulle et al. 2019). Alternatively, these hyperactive comets could have formed in the outermost regions of the solar nebula. Indeed, modeling shows that a non-monotonic dependence of the $\mathrm{D} / \mathrm{H}$ ratio in the solar nebula may be expected. The ratio would decrease again in the outer regions of the disk, because water molecules that underwent isotopic exchanges at high temperatures near the young star would have been transported outward during the early disk evolution (Yang et al. 2013). The anti-correlation between hyperactivity and nucleus size appears inconsistent with the first explanation as planetesimals near the snow line are expected to undergo rapid growth.

An alternative explanation is that the isotopic properties of water outgassed from the nucleus surface and icy grains may be different, owing to fractionation processes during the sublimation of water ice. The observed anti-correlation can be reproduced with two sources of water contributing to the measured water production rate and the active fraction: D-rich water molecules released from the nucleus and an additional source of D-poor water molecules from sublimating icy grains (see dash-dotted line in Fig. 2). Laboratory experiments on sam- ples of pure ice show small deuterium fractionation effects (Lécuyer et al. 2017). In experiments with water ice mixed with dust, the released water vapor is depleted in deuterium, explained by preferential adsorption of HDO on dust grains (Moores et al. 2012). This effect goes in the opposite direction to the observed trend, while conversely the VSMOW D/H value from subliming icy grains is likely representative of buried nucleus water ice. Alternatively, a non-steady-state regime of water ice sublimation could explain the factor 2-3 deuterium enrichment in water vapor released from the nucleus with respect to VSMOW (Podolak et al. 2002). However, this would occur in episodic time intervals along the comet orbit (Podolak et al. 2002), whereas the $\mathrm{HDO} / \mathrm{H}_{2} \mathrm{O}$ values measured for comet $67 \mathrm{P}$ at different periods are very uniform (Altwegg et al. 2017).

\section{Conclusion}

Enlarging the number of accurate $\mathrm{D} / \mathrm{H}$ measurements in both Jupiter-family and Oort cloud comets is required to better constrain the observed correlation. Taking these measurements from the ground is challenging. Nearly simultaneous spectroscopic observations of low-energy rotational lines of $\mathrm{H}_{2}^{18} \mathrm{O}$ and HDO in a matching field of view encompassing a large fraction of the coma using the GREAT spectrometer aboard SOFIA can play a key role in this endeavor. In this context, the next close apparition of comet 67P/Churyumov-Gerasimenko in 2021 November will offer an excellent opportunity to re-measure the $\mathrm{D} / \mathrm{H}$ ratio in this comet using spectroscopic techniques.

The understanding of the observed correlation calls for detailed investigations of the mechanisms leading to dust and chunk ejection, and cometary hyperactivity. Further experimental and modeling work on evaporative fractionation is also clearly needed, and may ultimately establish that all comets share the same Earth-like water $\mathrm{D} / \mathrm{H}$ ratio, with profound implications on the early solar system and the origin of the Earth's oceans.

Acknowledgements. Based on observations made with the NASA/DLR Stratospheric Observatory for Infrared Astronomy (SOFIA). SOFIA is jointly operated by the Universities Space Research Association, Inc. (USRA), under NASA contract NAS2-97001, and the Deutsches SOFIA Institut (DSI) under DLR contract 50 OK 0901 to the University of Stuttgart. GREAT is a development by the MPI für Radioastronomie and the KOSMA/Universität zu Köln, in cooperation with the DLR Institut für Optische Sensorsysteme, financed by the participating institutes, by the German Aerospace Center (DLR) under grants 50 OK 1102, 1103, and 1104, and within the Collaborative Research Centre 956, funded by the Deutsche Forschungsgemeinschaft (DFG). Mixers for Channel 1 of the 4GREAT (4G-1) instrument have been designed and developed by LERMA (Observatoire de Paris, CNRS, Sorbonne Université, Université de Cergy-Pontoise) in the framework of the Herschel/HIFI project, with funding from the CNES. Part of this research was carried out at the Jet Propulsion Laboratory, California Institute of Technology, under a contract with the National Aeronautics and Space Administration. We thank the SOFIA project office for their excellent support and for adapting the operations and engineering support to the visibility constraints of the comet. We thank J. Blum, M. Fulle, and A. Morbidelli for the useful discussions.

Note added in proof. After the present manuscript was accepted for publication, it was brought to our attention that the correlation between the active fraction and the nucleus size was independently found by Sosa \& Fernández (2011).

\section{References}

A'Hearn, M. F., Campins, H., Schleicher, D. G., \& Millis, R. L. 1989, ApJ, 347, 1155

A'Hearn, M. F., Belton, M. J. S., Delamere, W. A., et al. 2005, Science, 310, 258 Alexander, C. M. O. D., Bowden, R., Fogel, M. L., et al. 2012, Science, 337, 721 
Altwegg, K., Balsiger, H., Bar-Nun, A., et al. 2015, Science, 347, 1261952 Altwegg, K., Balsiger, H., Berthelier, J. J., et al. 2017, Phil. Trans. R. Soc. Ser. A, 375, 20160253

Bischoff, D., Gundlach, B., Neuhaus, M., \& Blum, J. 2019, MNRAS, 483, 1202

Biver, N., Bockelée-Morvan, D., Crovisier, J., et al. 1999, AJ, 118, 1850

Biver, N., Bockelée-Morvan, D., Boissier, J., et al. 2007, Icarus, 187, 253

Biver, N., Crovisier, J., Bockelée-Morvan, D., et al. 2012, A\&A, 539, A68

Biver, N., Moreno, R., Bockelée-Morvan, D., et al. 2016, A\&A, 589, A78

Bockelée-Morvan, D., Gautier, D., Lis, D., et al. 1998, Icarus, 133, 147

Bockelée-Morvan, D., Calmonte, U., Charnley, S., et al. 2015, Space Sci. Rev., 197, 47

Boissier, J., Groussin, O., Jorda, L., et al. 2013, A\&A, 557, A88

Brasser, R., \& Morbidelli, A. 2013, Icarus, 225, 40

Brownlee, D. E., Horz, F., Newburn, R. L., et al. 2004, Science, 304, 1764

Ceccarelli, C., Caselli, P., Bockelée-Morvan, D., et al. 2014, Protostars and Planets VI, 859

Combi, M. R., Mäkinen, J. T. T., Bertaux, J. L., \& Quémerais, E. 2005, Icarus, 177,228

Combi, M. R., Mäkinen, J. T. T., Bertaux, J. L., Quémerais, E., \& Ferron, S. 2019, Icarus, 317, 610

Cooke, R. J., Pettini, M., Jorgenson, R. A., Murphy, M. T., \& Steidel, C. C. 2014 ApJ, 781, 31

Cowan, J. J., \& A’Hearn, M. F. 1979, Moon and Planets, 21, 155

Deloule, E., Robert, F., \& Doukhan, J. C. 1998, Geoch. Cosmoch. Acta, 62, 3367

de Val-Borro, M., Hartogh, P., Crovisieret, J., et al. 2010, A\&A, 521, L50

Drouart, A., Dubrulle, B., Gautier, D., \& Robert, F. 1999, Icarus, 140, 129

Durán, C. A., Güsten, R., Risacher, C., et al. 2017, ISST Proc., 27

Feldman, P. D., Festou, M. C., Tozzi, P., \& Weaver, H. A. 1997, ApJ, 475, 829

Fernández, Y. R., Kelley, M. S., Lamy, P. L., et al. 2013, Icarus, 226, 1138

Fulle, M., Blum, J., Green, S. F., et al. 2019, MNRAS, 482, 3326

Geiss, J., \& Gloeckler, G. 1998, Space Sci. Rev., 84, 239

Guan, X., Stutzki, J., Graf, U. U., et al. 2012, A\&A, 542, L4

Gulkis, S., Allen, M., von Allmen, P., et al. 2015, Science, 347, aaa0709

Hallis, L. J. 2015, Science, 350, 795

Harmon, J. K., Ostro, S. J., Benner, L. A. M., et al. 1997, Science, 278, 1921
Hartogh, P., Lis, D. C., Bockelée-Morvan, D., et al. 2011, Nature, 478, 218

Herique, A., Kofman, W., Beck, P., et al. 2016, MNRAS, 462, S516

Heyminck, S., Graf, U. U., Güsten, R., et al. 2012, A\&A, 542, L1

Kelley, M. S. P., Lindler, D. J., Bodewits, D., et al. 2013, Icarus, 222, 634

Kelley, M. S. P., Lindler, D. J., Bodewits, D., et al. 2015, Icarus, 262, 187

Lamy, P. L., Toth, I., Fernández, Y. R., \& Weaver, H. A. 2004, Comets II, 1, 223

Lécuyer, C., Royer, A., Fourel, F., et al. 2017, Icarus, 285, 1

Lis, D. C., Keene, J., Young, K., et al. 1997, Icarus, 130, 355

Lis, D. C., Roueff, E., Gerin, M., et al. 2002, ApJ, 571, L55

Lis, D. C., Biver, N., Bockelée-Morvan, D., et al. 2013, ApJ, 774, L3

McCanta, M. C., Treiman, A. H., Dyar, M. D., et al. 2008, Geochem. Astrochim. Acta, 72, 1401

Moores, J. E., Brown, R. H., Lauretta, D. S., \& Smith, P. H. 2012, Planet. Sci., 1,2

O’Brien, D. P., Izidoro, A., Jacobson, S. A., Raymond, S. N., \& Rubie, D. C. 2018, Space Sci. Rev., 214, 47

Paganini, L., Mumma, M. J., Gibb, E. L., \& Villanueva, G. L. 2017, ApJ, 836, L25

Parise, B., Castets, A., Herbst, E., et al. 2004, A\&A, 416, 159

Pätzold, M., Andert, T. P., Hahn, M., et al. 2019, MNRAS, 483, 2337

Pickett, H. M., Poynter, R. L., Cohen, E. A., et al. 1998, J. Quant. Spectr. Rad. Transf., 60, 883

Podolak, M., Mekler, Y., \& Prialnik, D. 2002, Icarus, 160, 208

Protopapa, S., Sunshine, J. M., Feaga, L. M., et al. 2014, Icarus, 238, 191

Risacher, C., Güsten, R., Stutzk, J., et al. 2018, J. Astron. Instr., 7, 1840014

Schoonenberg, D., \& Ormel, C. W. 2017, A\&A, 602, A21

Sosa, A., \& Fernández, J. A. 2011, MNRAS, 416, 767

van Dishoeck, E. F., Bergin, E. A., Lis, D. C., et al. 2014, Protostars and Planets VI, 835

Westall, F. 2018, Space Sci. Rev., 214, 50

Yang, L., Ciesla, F. J., \& Alexander, C. M. D. 2013, Icarus, 226, 256

Zakharov, V., Bockelée-Morvan, D., Biver, N., et al. 2007, A\&A, 473, 303 


\section{Appendix A: Observations and modeling}

\section{A.1. Observations}

The observations of comet 46P/Wirtanen reported here were carried out using the GREAT heterodyne spectrometer (Heyminck et al. 2012) aboard SOFIA during five flights between 2018 December 14 and 20 UT, out of Palmdale, CA, USA. The instrument was operated in its upGREAT HFA/4GREAT (HFA/4G) configuration (Risacher et al. 2018; Durán et al. 2017), which allows simultaneous observations at five different frequencies. The lowest frequency band, 4G-1, was used for the observations reported here. The tuning setup and the basic instrument characteristics are summarized in Table A.1. Although several other transitions of interest were covered in the higherfrequency channels, only $\mathrm{OH}$ was detected at a low signal-to-noise ratio, and the other upper limits are not constraining owing to the much higher system temperatures at these frequencies.

The instrument was operated in double-beam chopped mode, with a chopper throw of $200^{\prime \prime}$, at a rate of $2.5 \mathrm{~Hz}$. The comet was tracked using an ephemeris based on the orbital solution 181-11 generated using JPL Horizons ${ }^{2}$. Pointing was established by the telescope operators directly on the optical core of the comet to an accuracy of 2-3". The local oscillator frequency was updated every $4 \mathrm{~min}$ according to the ephemeris, introducing a maximum velocity tracking error of about $0.002 \mathrm{~km} \mathrm{~s}^{-1}$. Prior to the flight series, the optical axis of the GREAT instrument had been aligned to the optical imagers by observations of Mars. The main beam coupling efficiencies, also determined from observations of Mars, and the diffraction limited half-power beam widths are listed in Table A.1. The observations were performed at flight altitudes between 40000 and 43000 feet. Atmospheric conditions were typical for late autumn flights out of Palmdale, CA, with a residual water vapor column of about $15-20 \mu \mathrm{m}$, which resulted in typical single-sideband system temperatures $T_{\text {sys }}$ of about $300 \mathrm{~K}$ (Table A.1).

The calibration at the frequencies of the $\mathrm{HDO}$ and $\mathrm{H}_{2}^{18} \mathrm{O}$ lines is challenging. Locally, the lines are affected by the proximity of their rather narrow telluric counterparts, shifted by about \pm 3 $\mathrm{km} \mathrm{s}^{-1}$ at the time of observation. Moreover, the transmission at the $\mathrm{H}_{2}^{18} \mathrm{O}$ frequency is strongly affected by the broad absorption of the nearby telluric $\mathrm{H}_{2}^{16} \mathrm{O}$ line at $557 \mathrm{GHz}$ (50\% transmission only). The spectra were corrected for atmospheric losses following the usual calibration scheme (Guan et al. 2012) based on two load signals (one at ambient temperature and one at a cold temperature) to determine the instrument gain and a blank sky signal (chopper off phase), to which the atmospheric model was fit in order to correct the observed signal to outside the atmosphere. The resulting calibration uncertainties at the frequencies of 509 and $547 \mathrm{GHz}$ are $10 \%$ and $15 \%$, respectively.

Calibrated spectra provided by the instrument pipeline were further reduced and analyzed using the IRAM Gildas software ${ }^{3}$. A linear baseline was first subtracted from each scan and the resulting spectra were then averaged with $1 / \sigma^{2}$ weighting by radiometric noise. The observing $\log$ is shown in Table A.2. The heliocentric and geocentric distance of the comet changed only slightly during the period of the observations, with average values of 1.058 and $0.079 \mathrm{au}$, respectively. The total on-source integration time is 64 and $150 \mathrm{~min}$ for $\mathrm{H}_{2}^{18} \mathrm{O}$ and $\mathrm{HDO}$, respectively. There is some evidence for day-to-day variations in the $\mathrm{H}_{2}^{18} \mathrm{O}$ line intensity (the intensity on December 19 UT seems lower compared to the other days; see inset in the upper panel of

\footnotetext{
2 https://ssd.jpl.nasa.gov/horizons.cgi
}

http: //WwW . iram. fr/IRAMFR/GILDAS
Table A.1. Instrument tuning and performance.

\begin{tabular}{cccccc}
\hline \hline Transition & $\begin{array}{c}v \\
(\mathrm{GHz})\end{array}$ & $\begin{array}{c}\mathrm{IF}_{\mathrm{c}} \\
(\mathrm{GHz})\end{array}$ & $\begin{array}{c}\mathrm{T}_{\text {sys }} \\
(\mathrm{K})\end{array}$ & $\begin{array}{c}\Theta \\
\left({ }^{\prime \prime}\right)\end{array}$ & $\eta_{\mathrm{mb}}$ \\
\hline $\mathrm{H}_{2}^{18} \mathrm{O}\left(1_{1,0}-1_{0,1}\right)$ & 547.676440 & $5.4 \mathrm{U}$ & 317 & 50.3 & 0.63 \\
$\mathrm{HDO}\left(1_{1,0}-1_{0,1}\right)$ & 509.292420 & $6.2 / 5.45 \mathrm{U}$ & 290 & 54.1 & 0.63 \\
\hline
\end{tabular}

Notes. Entries in the table are molecular transition, rest frequency (from JPL Molecular Spectroscopy Database; Pickett et al. 1998), intermediate frequency and sideband (carefully chosen by balancing between the atmospheric transmission in the upper and lower sidebands, the receiver performance, and possible line contamination from the image sideband), single sideband system temperature (average across the intermediate frequency band), FWHM beam width, and main beam efficiency.

Fig. 1). However, the signal-to-noise ratio in the spectra obtained on the individual flights is limited. Since both $\mathrm{HDO}$ and $\mathrm{H}_{2}^{18} \mathrm{O}$ were observed on each flight and we do not expect day-to-day variations in the isotopic ratio, we use the average spectra in the subsequent analysis.

\section{A.2. Modeling}

To convert the observed line intensities into molecular production rates, we used an excitation model similar to that used in our earlier Herschel studies (Hartogh et al. 2011; Lis et al. 2013). We computed several models with different assumptions for the collisions with electrons. We used electron density factors $x_{\text {ne }}=0,0.1$, and 0.2 and a contact surface scaling factor $X_{\mathrm{re}}=0.5$ (Zakharov et al. 2007). We used a variable temperature model with a temperature of $80 \mathrm{~K}$ out to a distance of $270 \mathrm{~km}$ (corresponding to approximately $10 \%$ of the field of view), followed by a linear decrease to $12 \mathrm{~K}$ at a distance of $630 \mathrm{~km}$, and a constant temperature at larger distances. A similar temperature profile provided a good fit to the observed spatial distribution of multiple water emission lines observed in comet 103P/Hartley. We also computed models with constant temperatures of 40 and $60 \mathrm{~K}$, consistent with ground-based methanol observations carried out by members of our team using the IRAM $30 \mathrm{~m}$ telescope. The maximum difference in the isotopic ratios retrieved using the various models is $15 \%$. In our analysis we used the average molecular production rates provided by the various models with a conservative modeling uncertainty of $10 \%$.

The observed line intensities lead to average $\mathrm{HDO}$ and $\mathrm{H}_{2}^{18} \mathrm{O}$ production rates of $(2.5 \pm 0.9) \times 10^{24} \mathrm{~s}^{-1}$ and $(1.5 \pm 0.3) \times 10^{25} \mathrm{~s}^{-1}$, respectively, where the uncertainties include the statistical and calibration uncertainties, and a $10 \%$ modeling uncertainty, combined in quadrature. Assuming a ${ }^{16} \mathrm{O} /{ }^{18} \mathrm{O}$ isotopic ratio of $500 \pm$ 50 , we derive $\mathrm{H}_{2}^{16} \mathrm{O}$ production rate of $(7.7 \pm 1.5) \times 10^{27} \mathrm{~s}^{-1}$. The resulting $\mathrm{D} / \mathrm{H}$ ratio in water, $(1.61 \pm 0.65) \times 10^{-4}$, is close to the Earth's ocean value. The uncertainty includes a $10 \%$ uncertainty for the ${ }^{16} \mathrm{O} /{ }^{18} \mathrm{O}$ isotopic ratio, combined in quadrature with the statistical, calibration, and modeling uncertainties.

\section{A.3. Computations of the active fraction}

To compute the active fractional area, we used the sublimation model of Cowan \& A'Hearn (1979) for a rotational pole pointed at the Sun, which is identical to both the non-rotating case and to the case of zero thermal inertia. This model is appropriate, as cometary nuclei have low thermal inertia (Gulkis et al. 2015). We use tabulated values for the average water sublimation rate per surface unit, $Z$, as a function of the heliocentric distance, $r_{\mathrm{h}}$. Calculations are carried out for a Bond albedo of 0.05 and $100 \%$ 
Table A.2. SOFIA observations of comet 46P/Wirtanen.

\begin{tabular}{cccccccc}
\hline \hline Flight & $\begin{array}{c}\mathrm{UT} \mathrm{time} \\
(\mathrm{hr})\end{array}$ & $\begin{array}{c}r_{h} \\
(\mathrm{au})\end{array}$ & $\begin{array}{c}\Delta \\
(\mathrm{au})\end{array}$ & $\begin{array}{c}t\left(\mathrm{H}_{2}^{18} \mathrm{O}\right) \\
(\mathrm{min})\end{array}$ & $\begin{array}{c}\sigma\left(\mathrm{H}_{2}^{18} \mathrm{O}\right) \\
(\mathrm{mK})\end{array}$ & $\begin{array}{c}t(\mathrm{HDO}) \\
(\mathrm{min})\end{array}$ & $\begin{array}{c}\sigma(\mathrm{HDO}) \\
(\mathrm{mK})\end{array}$ \\
\hline 1 & Dec 14, 4.89-7.47 & 1.056 & 0.079 & 16.5 & 80 & 29.2 & 43 \\
2 & Dec 17, 7.56-9.68 & 1.057 & 0.078 & 7.2 & 125 & 30.8 & 38 \\
3 & Dec 18, 9.59-12.17 & 1.058 & 0.078 & 13.8 & 112 & 30.3 & 37 \\
4 & Dec 19, 9.78-12.00 & 1.059 & 0.079 & 14.9 & 85 & 25.6 & 42 \\
5 & Dec 20,9.83-12.33 & 1.060 & 0.081 & 11.6 & 105 & 34.1 & 31 \\
\hline
\end{tabular}

Notes. Entries in the table are flight number, UT range in hours, average heliocentric and geocentric distance of the comet (au) as given by the ephemeris, total on-source integration time (minutes), and the resulting rms noise level in a $0.14 \mathrm{~km} \mathrm{~s}^{-1}$ velocity channel (mK) for $\mathrm{H}_{2}^{18} \mathrm{O}$ and $\mathrm{HDO}$. The comet reached perihelion on December 12 at 22:20 UT and made the closest approach to the Earth on December 16 at 13:06 UT. At a 0.08 au geocentric distance, the field of view on the comet was about $3000 \mathrm{~km}$.

Table A.3. Comet properties and derived active fractions.

\begin{tabular}{lccccc}
\hline \hline Comet & $\begin{array}{c}q^{(a)} \\
(\mathrm{au})\end{array}$ & $\begin{array}{c}r_{\mathrm{N}}{ }^{(b)} \\
(\mathrm{km})\end{array}$ & $\mathrm{D} / \mathrm{H}^{(c)}$ & $\begin{array}{c}Q\left(\mathrm{H}_{2} \mathrm{O}\right) \\
\mathrm{s}^{-1}\end{array}$ & Active fraction ${ }^{(e)}$ \\
\hline $1 \mathrm{P}$ & 0.59 & 5.50 & $(3.10 \pm 0.40) \times 10^{-4}$ & $1.5 \times 10^{30}$ & $(3.3 \pm 1.0) \times 10^{-1}$ \\
$8 \mathrm{P}$ & 1.03 & 3.10 & $(4.09 \pm 1.45) \times 10^{-4}$ & $0.3 \times 10^{29}$ & $(8.5 \pm 2.6) \times 10^{-2}$ \\
$45 \mathrm{P}$ & 0.53 & $0.43 \pm 0.10$ & $<2.00 \times 10^{-4}$ & $0.5 \times 10^{29}$ & $(1.6 \pm 0.6) \times 10^{0}$ \\
$46 \mathrm{P}$ & 1.05 & $0.63 \pm 0.04$ & $(1.61 \pm 0.65) \times 10^{-4}$ & $1.9 \times 10^{28}$ & $(1.2 \pm 0.4) \times 10^{0}$ \\
$67 \mathrm{P}$ & 1.24 & 2.00 & $(5.30 \pm 0.70) \times 10^{-4}$ & $0.9 \times 10^{28}$ & $(8.3 \pm 2.5) \times 10^{-2}$ \\
$103 \mathrm{P}$ & 1.06 & 0.58 & $(1.61 \pm 0.24) \times 10^{-4}$ & $1.0 \times 10^{28}$ & $(7.2 \pm 2.2) \times 10^{-1}$ \\
$\mathrm{C} / 1995 \mathrm{O} 1$ & 0.91 & $30.00 \pm 10.00$ & $(3.30 \pm 0.80) \times 10^{-4}$ & $1.7 \times 10^{31}$ & $(3.4 \pm 1.5) \times 10^{-1}$ \\
$\mathrm{C} / 1996 \mathrm{~B} 2$ & 0.23 & $1.20 \pm 0.25$ & $(1.85 \pm 0.60) \times 10^{-4}$ & $0.9 \times 10^{31}$ & $(6.1 \pm 2.2) \times 0^{0}$ \\
$\mathrm{C} / 2009 \mathrm{P} 1$ & 1.55 & $<5.60$ & $(2.06 \pm 0.22) \times 10^{-4}$ & $1.9 \times 10^{29}$ & $(3.9 \pm 1.2) \times 10^{-1}$ \\
2P & 0.33 & $2.40 \pm 0.30$ & & $0.8 \times 10^{29}$ & $(2.9 \pm 0.9) \times 10^{-2}$ \\
9P & 1.51 & 2.72 & & $0.8 \times 10^{28}$ & $(6.3 \pm 1.9) \times 10^{-2}$ \\
$10 \mathrm{P}$ & 1.42 & $5.90 \pm 0.70$ & & $2.2 \times 10^{28}$ & $(3.2 \pm 1.0) \times 10^{-2}$ \\
$19 \mathrm{P}$ & 1.36 & 2.40 & & $0.5 \times 10^{29}$ & $(4.1 \pm 1.2) \times 10^{-1}$ \\
21P & 1.04 & $1.82 \pm 0.05$ & & $0.4 \times 10^{29}$ & $(2.7 \pm 0.8) \times 10^{-1}$ \\
$41 \mathrm{P}$ & 1.05 & 0.70 & & $1.1 \times 10^{28}$ & $(5.5 \pm 1.6) \times 10^{-1}$ \\
$55 \mathrm{P}$ & 0.98 & $1.84 \pm 0.15$ & & $0.5 \times 10^{29}$ & $(3.1 \pm 1.0) \times 10^{-1}$ \\
$73 \mathrm{P}$ & 0.94 & $1.10 \pm 0.03$ & & $0.9 \times 10^{29}$ & $(1.4 \pm 0.4) \times 10^{0}$ \\
81P & 1.60 & 2.10 & & $1.0 \times 10^{28}$ & $(1.5 \pm 0.5) \times 10^{-1}$ \\
96P & 0.12 & $3.20 \pm 0.20$ & & $0.4 \times 10^{30}$ & $(1.2 \pm 0.4) \times 10^{-2}$ \\
\hline
\end{tabular}

Notes. ${ }^{(a)}$ Perihelion distance. ${ }^{(b)}$ Nucleus effective radius. See references in Combi et al. (2019) and Sect. A.3. For spacecraft and radar data, the error was assumed to be insignificant. ${ }^{(c)} \mathrm{D} / \mathrm{H}$ in water. References in the review of Bockelée-Morvan et al. (2015), except for 67P (Altwegg et al. 2015), C/1996B2 (this work), and 46P (this work). ${ }^{(d)}$ Water production rate at perihelion distance. From SWAN measurements (Combi et al. 2019), except for 1P (Feldman et al. 1997), 9P (Biver et al. 2007), 10P (Biver et al. 2012), and 81P (de Val-Borro et al. 2010). See Sect. A.3. ${ }^{(e)}$ Active fraction at perihelion distance.

infrared emissivity. At $r_{\mathrm{h}}=1 \mathrm{au}, Z=3.6 \times 10^{21} \mathrm{~mol} \mathrm{~s}^{-1} \mathrm{~m}^{-2}$. The active area (AA) is obtained by dividing the water production rate by $Z$, and the active fractional area is obtained by dividing AA by the nucleus surface area $\left(4 \pi r_{\mathrm{N}}^{2}\right.$, where $r_{\mathrm{N}}$ is the effective nucleus radius). We note that the derived active areas only provide a crude estimation of the ice exposed to the solar radiation, because the utilized sublimation model is simplistic. For example, the active fractions derived here differ by a large but constant factor from those computed assuming rapidly rotating nuclei (Combi et al. 2019).

We consider water production rates $Q\left(\mathrm{H}_{2} \mathrm{O}\right)$ derived from Lyman- $\alpha$ observations by the SWAN instrument aboard SOHO (Combi et al. 2019). Over $90 \%$ of the observed hydrogen atoms are produced by $\mathrm{H}_{2} \mathrm{O}$ or its photodissociation product $\mathrm{OH}$. We use the reported absolute water production rates at $r_{\mathrm{h}}=1 \mathrm{au}$, and pre-/post-perihelion power laws with $r_{\mathrm{h}}$ to derive water production rates at perihelion by averaging the production rates deduced from pre- and post-perihelion laws. For some shortperiod comets, the SWAN survey includes multiple apparitions (e.g., 1997 and 2002 for 46P). In this case, we used the average results for multiple apparitions (Table 4 of Combi et al. 2019). The SWAN survey does not include comet 1P/Halley, for which we assumed $\mathrm{Q}\left(\mathrm{H}_{2} \mathrm{O}\right)=5 \times 10^{29} \mathrm{~s}^{-1}\left(r_{\mathrm{h}}=1.0 \mathrm{au}\right.$, with a $r_{\mathrm{h}}^{-2}$ variation for $\mathrm{Q}\left(\mathrm{H}_{2} \mathrm{O}\right)$; Feldman et al. 1997). Water production rates used to compute the active fractions are listed in Table A.3 (values at perihelion). For consistency, we did not consider the water production rate of 46P derived from the SOFIA 2018 observations (Sect. A.1), which is about a factor of two lower than the SWAN value (Table A.3), possibly because of the smaller projected field of view for this close apparition. This trend between aperture size and water production is observed for hyperactive comets.

To study how the active fraction correlates with the nucleus size, we added to our sample ten short-period comets with well-characterized water production rates and nucleus sizes: $2 \mathrm{P} /$ Encke, 9P/Tempel 1, 10P/Tempel 2, 19P/Borrelly, 21P/GiacobiniZinner, 41P/Tuttle-Giacobini-Kresak, 55P/Tempel-Tuttle, 73P/ Schwassmann-Wachmann 3, 81P/Wild 2, and 96P/Machholz 1. Water production rates are from Combi et al. (2019), except for 9P, $10 \mathrm{P}$, and 81P, for which we used measurements from Biver et al. (2007, 2012), and de Val-Borro et al. (2010). Most of the nucleus 


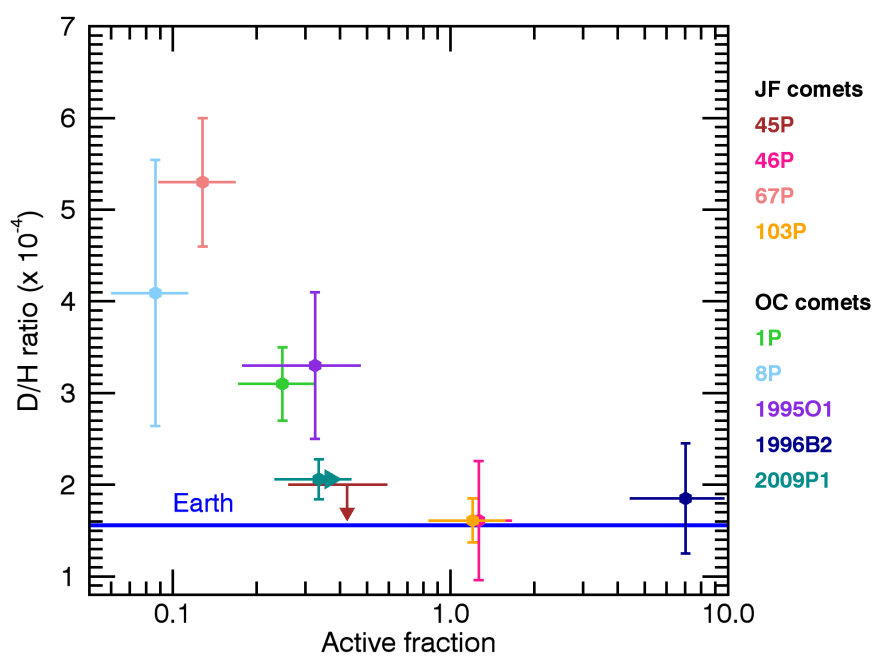

Fig. A.1. D/H ratio in cometary water as a function of the active fraction computed based on the water production rates at 1 au from the Sun. The color of each symbol indicates a comet; see legend at right, where the dynamical class is also indicated: Oort cloud (OC) or short-period Jupiter-family (JF) comets. The uncertainties on the active fraction (horizontal bars) include a $30 \%$ uncertainty on the water production rates (Combi et al. 2019) and the uncertainty on the nucleus size.

sizes are given in Combi et al. (2019). For C/199501 (HaleBopp), we used a nucleus radius of $30 \mathrm{~km}$ (Lamy et al. 2004). For C/1996 B2 (Hyakutake), we used a value of $1.2 \mathrm{~km}$ (Harmon et al. 1997). For 9P, 10P, and 81P the effective nucleus radii are $2.72,5.9 \mathrm{~km}$, and $2.1 \mathrm{~km}$, respectively (A'Hearn et al. 1989, 2005; Brownlee et al. 2004). Comet properties and derived active fractions at perihelion are summarized in Table A.3.

There are strong biases in this study. Comets with low water production rates (below $5 \times 10^{27} \mathrm{~s}^{-1}$ at $1 \mathrm{au}$ ) are not considered. In addition, small nuclei are largely underrepresented, considering estimates of the size distribution of short-period comets (Fernández et al. 2013). Therefore, our sample does not include small comets with low active fractions, which may be present in the population of short-period comets, because their surface has been heavily mantled by refractory dust. However, large $\left(r_{\mathrm{N}}>1 \mathrm{~km}\right)$ comets are well represented in our sample.

Figure A. 2 shows the active fraction computed at perihelion plotted as a function of perihelion distance. There is no significant correlation between these two quantities. However, the two short-period comets $2 \mathrm{P}$ and $96 \mathrm{P}$ with small perihelion distances ( 0.34 and $0.12 \mathrm{au}$, respectively) have a low active fraction, which may be related to a gradual decrease in the ice-to-refractory ratio in subsurface layers (Combi et al. 2019). We also looked for a possible dependence of the $\mathrm{D} / \mathrm{H}$ ratio on the nucleus size, but do not find a statistically significant correlation between these two quantities.

\section{A.4. D/H ratio in comet C/1996 B2 (Hyakutake)}

The HDO production rate in comet Hyakutake was measured to be $(1.20 \pm 0.28) \times 10^{26} \mathrm{~s}^{-1}$, averaging data obtained on March 23.5 and 24.5 UT, 1996 with the Caltech Submillimeter Observatory (CSO; Bockelée-Morvan et al. 1998). A D/H ratio of $(2.9 \pm 1.0) \times 10^{-4}$ was derived using a water production rate of $(2.1 \pm 0.5) \times 10^{29} \mathrm{~s}^{-1}$, corresponding to the average of reported measurements using observations of $\mathrm{H}_{2} \mathrm{O}$ (IR), $\mathrm{OH}$ (UV, radio), H Lyman- $\alpha$, and OI (optical). An updated analysis of the H Lyman- $\alpha$ SWAN observations (Combi et al.

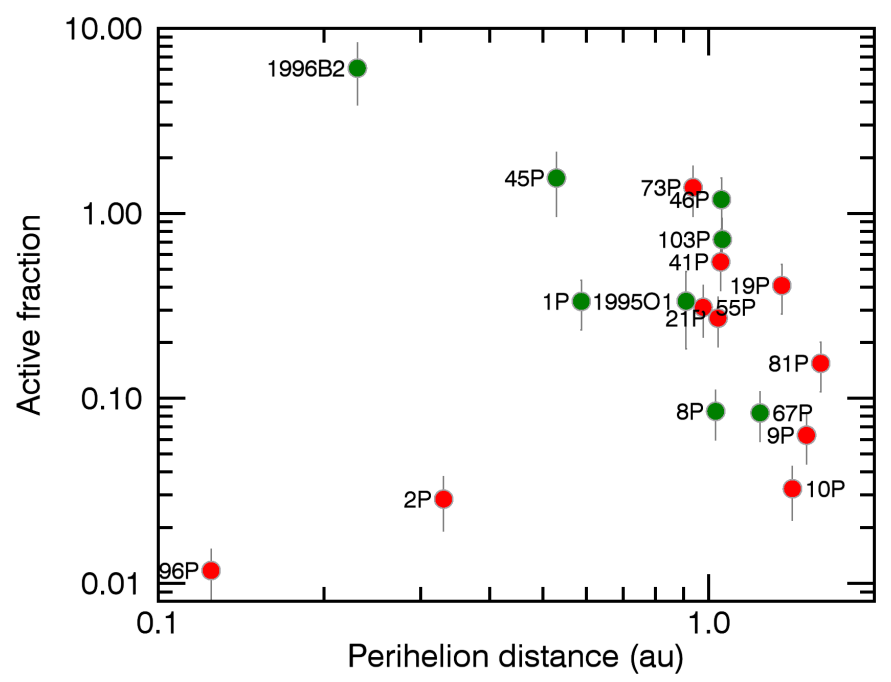

Fig. A.2. Active fraction at perihelion as a function of perihelion distance for a sample of 18 comets. Green symbols refer to comets for which the $\mathrm{D} / \mathrm{H}$ ratio in water has been measured. The uncertainties on the active fraction (vertical bars) include a $30 \%$ uncertainty on the water production rates (Combi et al. 2019) and the uncertainty on the nucleus size.

2005) indicates a higher water production rate than that adopted in Bockelée-Morvan et al. (1998). Combi et al. (2005) compare SWAN retrievals to other $Q\left(\mathrm{H}_{2} \mathrm{O}\right)$ measurements and conclude that there is a relatively good agreement, taking into account modeling induced differences. Using the daily tabulated $Q\left(\mathrm{H}_{2} \mathrm{O}\right)$ values in Table 2 of Combi et al. (2005) and using interpolation, we derive $Q\left(\mathrm{H}_{2} \mathrm{O}\right)=3.23 \times 10^{29} \mathrm{~s}^{-1}$ during the time of the HDO observations. Assuming a $25 \%$ uncertainty for $Q\left(\mathrm{H}_{2} \mathrm{O}\right)$ (Combi et al. 2005), the revised $\mathrm{D} / \mathrm{H}$ ratio in comet Hyakutake is then $(1.85 \pm 0.6) \times 10^{-4}$.

HDO was detected in comet Hyakutake during an outburst. We therefore re-evaluated the $\mathrm{HDO} / \mathrm{H}_{2} \mathrm{O}$ production rate ratio using the methanol lines observed in the same spectrum as HDO and the re-evaluated $\mathrm{CH}_{3} \mathrm{OH}$ average abundance relative to water outside the outburst period. Using water production rates from Combi et al. (2005) and the methanol production rates derived from JCMT, PdBi, and CSO observations before and after the 19-24 March outburst period (Biver et al. 1999; Lis et al. 1997), we find $Q\left(\mathrm{CH}_{3} \mathrm{OH}\right) / \mathrm{Q}\left(\mathrm{H}_{2} \mathrm{O}\right)=0.013 \pm 0.003$. The computation of the HDO and methanol production rates from the CSO data of 23.5 and 24.5 March yields $Q(\mathrm{HDO}) / Q\left(\mathrm{CH}_{3} \mathrm{OH}\right)=$ $0.026 \pm 0.005$. Hence, we infer $Q(\mathrm{HDO}) / Q\left(\mathrm{H}_{2} \mathrm{O}=(3.4 \pm 1) \times 10^{-4}\right.$ and consequently $\mathrm{D} / \mathrm{H}=(1.7 \pm 0.5) \times 10^{-4}$, in agreement with the value derived above.

\section{A.5. D/H ratio in comet C/2014 Q2 (Lovejoy)}

A $\mathrm{D} / \mathrm{H}$ ratio in water of $(1.4 \pm 0.4) \times 10^{-4}$ was measured in comet C/2014 Q2 (Lovejoy) based on the ground-based detection of a millimeter HDO line (Biver et al. 2016). Instead, infrared observations undertaken with a much smaller field of view (Paganini et al. 2017) yield $\mathrm{D} / \mathrm{H}=(3.02 \pm 0.87) \times 10^{-4}$. The inconsistency between the two values, which is marginal when considering the uncertainties of the two measurements, can be explained if this comet is a hyperactive comet. The VSMOW value measured in the millimeter would characterize water subliming from grains, whereas the value obtained in the IR would sample mainly water released directly from the nucleus. The size of the nucleus of this comet is currently unknown, so the active fraction cannot be computed. 